\title{
TRIGGERED STAR FORMATION AND THE CREATION OF THE SUPERGIANT H I SHELL IN IC 2574
}

\author{
Daniel R. Weisz ${ }^{1}$, Evan D. Skillman ${ }^{1}$, John M. Cannon ${ }^{2}$, Fabian Walter $^{3}$, Elias Brinks ${ }^{4}$, JÜRGen OtT $^{5,6,8}$, and Andrew \\ E. DOLPHIN ${ }^{7}$ \\ ${ }^{1}$ Astronomy Department, University of Minnesota, Minneapolis, MN 55455; USA; dweisz@astro.umn.edu, skillman@astro.umn.edu \\ ${ }^{2}$ Department of Physics \& Astronomy, Macalester College, 1600 Grand Avenue, St. Paul, MN 55125, USA; jcannon@macalester.edu \\ ${ }^{3}$ Max-Planck-Institut für Astronomie, Königstuhl 17, D-69117 Heidelberg, Germany; walter@mpia.de \\ ${ }^{4}$ Centre for Astrophysics Research, University of Hertfordshire, Hatfield AL10 9AB, UK; E.Brinks@herts.ac.uk \\ ${ }^{5}$ National Radio Observatory, 520 Edgemont Road, Charlottesville, VA 22903, USA; jott@nrao.edu \\ ${ }^{6}$ California Institute of Technology, 1200 E. California Blvd., Caltech Astronomy 105-24, Pasadena, CA 91125, USA \\ ${ }^{7}$ Raytheon Company, 1151 E Hermans Rd, Tucson, AZ 85756, USA; adolphin@ raytheon.com \\ Received 2008 November 17; accepted 2008 December 10; published 2009 January 7
}

\begin{abstract}
Based on deep imaging from the Advanced Camera for Surveys aboard the Hubble Space Telescope (HST), we present new evidence that stellar feedback created a $\sim 1 \mathrm{kpc}$ supergiant $\mathrm{H}$ I shell (SGS) and triggered star formation (SF) around its rim in the M81 Group dwarf irregular galaxy IC 2574. Using photometry of the resolved stars from the HST images, we measure the star formation history of a region including the SGS, focusing on the past $500 \mathrm{Myr}$, and employ the unique properties of blue helium-burning stars to create a movie of SF in the SGS. We find two significant episodes of SF inside the SGS from 200-300 Myr and $25 \mathrm{Myr}$ ago. Comparing the timing of the SF events to the dynamic age of the SGS and the energetics from the H I and SF, we find compelling evidence that stellar feedback is responsible for creating the SGS and triggering secondary SF around its rim.
\end{abstract}

Key words: galaxies: dwarf - galaxies: individual (IC 2574) - galaxies: irregular - galaxies: ISM - stars: formation

\section{INTRODUCTION}

Vigorous star formation (SF) is one mechanism thought to be responsible for the creation of the holes and shells observed in the interstellar medium (ISM) of a wide variety of galaxies. Following a strong episode of SF, energy input into the ISM from young massive stars (e.g., stellar winds, Type II supernovae) can provide sufficient mechanical energy to create these holes and shells and may even trigger secondary SF around the edges (e.g., Tenorio-Tagle \& Bodenheimer 1988; Puche et al. 1992). Although observations of young stellar clusters inside these ISM features confirm that this is one possible scenario, a majority of holes and shells do not contain associated bright young clusters (e.g., van der Hulst 1996; Rhode et al. 1999; Pasquali et al. 2008). One explanation is that only a select few ISM holes and shells have been observed with sufficient photometric depth to detect the associated clusters. Alternatively, a number of competing theories suggest the origins of holes and shells in the ISM are from mechanisms other than stellar feedback, e.g., high-velocity cloud collisions (e.g., Kamphuis et al. 1991), interactions (e.g., Bureau et al. 2004), gamma-ray bursts (e.g., Loeb \& Perna 1998), and stochastic SF propagation (Harris \& Zaritsky 2008).

Dwarf irregular galaxies (dIs) provide excellent environments in which we can study the creation mechanisms of these ISM features. Larger galaxies exhibit differential rotation and sheer, which can destroy the holes and shells in the ISM on timescales as short as $10^{7}$ year. However, dIs are typically solid body rotators (e.g., Skillman et al. 1988; Skillman 1996), which allows features in the ISM to grow to large sizes $(\sim 1 \mathrm{kpc})$ and stay coherent over longer timescales (e.g., Walter \& Brinks 1999).

Nearby dIs are particularly convenient probes of the interplay between SF and the ISM, because we can directly compare the resolved stellar populations to the H I component to better understand mechanisms of feedback. At a distance of $\sim 4 \mathrm{Mpc}$ (Karachentsev et al. 2002), IC 2574, an M81 Group dI, contains

\footnotetext{
8 Jürgen Ott is a Jansky Fellow of the National Radio Astronomy Observatory.
}

a "supergiant shell" (SGS) (denoted by the red ellipse in panels (a) and (b) of Figure 1; Walter et al. 1998; Walter \& Brinks 1999) and is an excellent candidate for specifically studying both the formation of the SGS and subsequent triggered SF around its rim. Walter \& Brinks (1999) classify the SGS as an ellipse $(\sim 1000 \times 500 \mathrm{pc})$; however, note that many of the holes and shells in IC 2574 are elongated due to projection effects (the de-projected diameter is $\sim 700 \mathrm{pc}$ ). They further note there is some evidence that the SGS is a superposition of two merged shells (Walter \& Brinks 1999), which could account for its elliptical shape. In addition to the shape, the expansion of the SGS is somewhat uncertain. Walter \& Brinks (1999) measure an H I expansion velocity of $25 \mathrm{~km} \mathrm{~s}^{-1}$ from a break in the PV diagram, which is only an indirect measure of expansion, and in fact is consistent with stalled expansion as well. We discuss the implications of these scenarios in Section 4. UV observations show a young stellar cluster $(\sim 11$ Myr old; Stewart \& Walter 2000) inside the SGS, but no $\mathrm{H} \alpha$ emission is observed from the interior. Around the rim, strong $\mathrm{H} \alpha$ (and mid-IR) emission is observed (see panel (c) of Figure 1, e.g., Walter et al. 1998; Cannon et al. 2005), indicating SF at these locations within the past $\sim 5$ Myr.

In this Letter, we use deep Hubble Space Telescope/Advanced Camera for Surveys (HST/ACS) imaging of the resolved stellar populations of the SGS to measure its recent star formation history (SFH) $(<500 \mathrm{Myr})$. We use the unique properties of resolved blue helium-burning stars $(\mathrm{BHeBs})$ to trace the spatial locations of the SF episodes, resulting in a movie that shows how SF propagated in the vicinity of the SGS over the past 500 Myr. From these lines of evidence, we are able to directly explore the SF event(s) responsible for the creation of the SGS and triggering of secondary SF.

\section{OBSERVATIONS AND PHOTOMETRY}

We used HST/ACS to observe the region of IC 2574 containing the SGS on 2004 February 6 in three wideband filters 


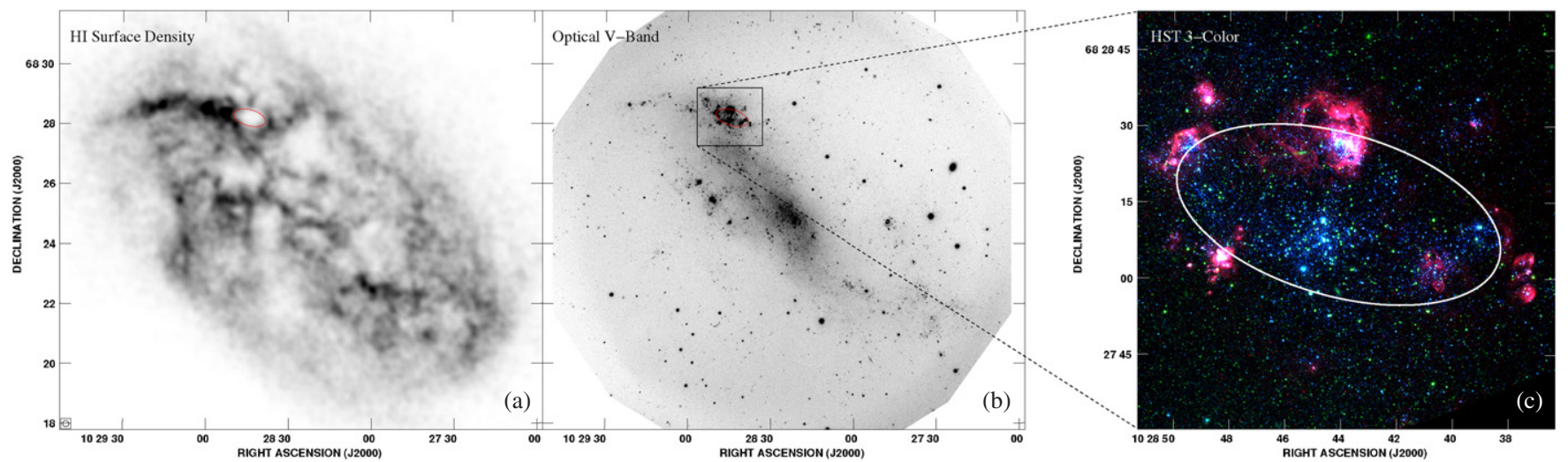

Figure 1. (a) H I image (Walter \& Brinks 1999), (b) optical $V$-band (Walter \& Brinks 1999), and (c) color ACS image of the SGS region in IC 2574 we use for analysis in this Letter. The ellipse in each image is the outline of the SGS and is possibly elliptical due to projection effects. It has a de-projected diameter of $\sim 700$ pc.

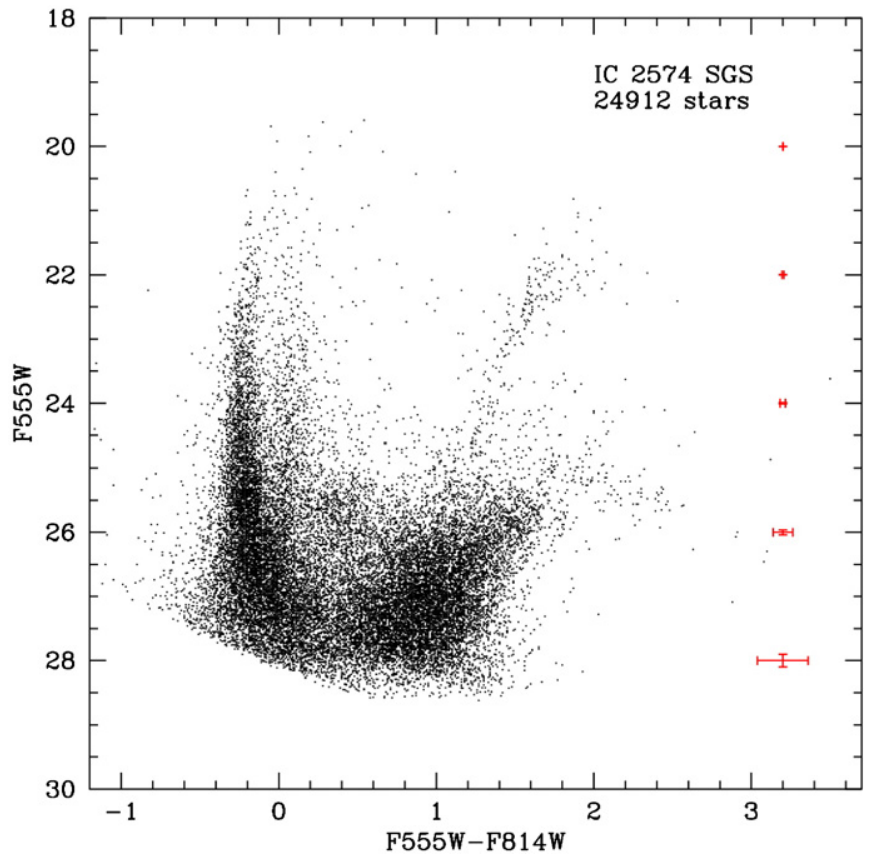

Figure 2. HST/ACS CMD of the SGS region (panel (c) in Figure 1) corrected for foreground extinction.

F435W (B), F555W ( $V)$, and F814W (I). The images were processed using the $H S T$ pipeline and photometry was extracted using DOLPHOT, a version of HSTPHOT (Dolphin 2000) with an ACS specific module. To account for photometric completeness, we performed artificial star tests. After filtering out cosmic rays, hot pixels, and extended sources from the photometry, we had 253,736 well measured stars in the ACS field of view. Subsequently, we isolated the SGS region and found 24,912 well measured stars in the F555W and F814W filters. The limiting magnitude of our photometry in this region is $M_{V} \sim 0$, which gives us excellent leverage on SF in the past $\sim 1$ Gyr (Gallart et al. 2005; Weisz et al. 2008). The photometry was carried out as part of a larger study of M81 Group dIs, and full details of the observations, photometry, and completeness can be found in Weisz et al. (2008).

\section{THE STAR FORMATION HISTORY}

We measured the SFH of the SGS region (the entirety of panel (c) in Figure 1) of IC 2574 from the HST/ACS based color-

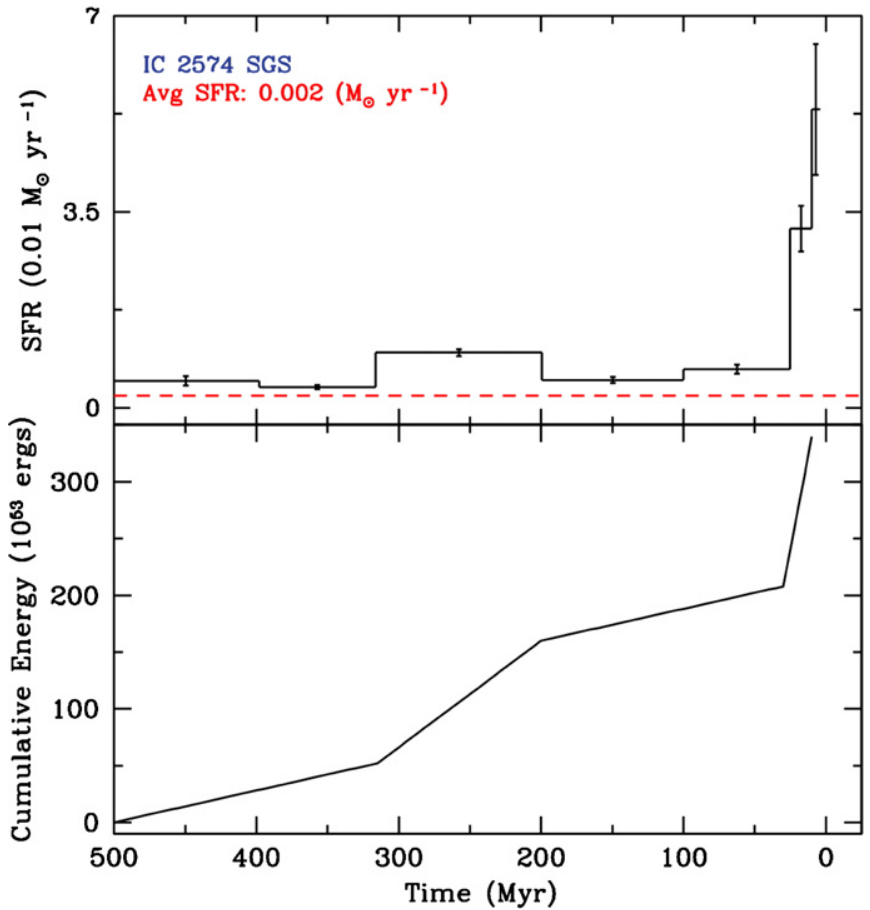

Figure 3. Top: the SFH of the SGS region over the past $500 \mathrm{Myr}$. The red dashed line indicates the SFR of the SGS averaged over the lifetime of the galaxy. Bottom: the cumulative energy from SF in the SGS over the past $500 \mathrm{Myr}$ calculated with STARBURST99.

magnitude diagram (CMD) using the code of Dolphin (2002). This method constructs synthetically generated CMDs, using the stellar evolution models of Marigo et al. (2008), and compares them to the observed CMD using a maximum likelihood technique. To obtain this solution, we used a combination of fixed (e.g., binary fraction, initial mass function) and searchable (e.g., distance, extinction) parameters. For consistency, we set all our parameters to the values found for IC 2574 listed in Table 2 of Weisz et al. (2008). To quantify the uncertainties of the SFH, we added the systematic uncertainties from the isochrones and the statistical uncertainties from Monte Carlo tests in quadrature (see Weisz et al. 2008 for details).

We used the CMD of the SGS region (see Figure 2) as input into the SFH code. The SFH of the SGS region over the past $500 \mathrm{Myr}$ is shown in the top panel of Figure 3. For reference, we show the star formation rate (SFR) of the SGS region averaged over the lifetime of the galaxy as the red dashed line. We see 


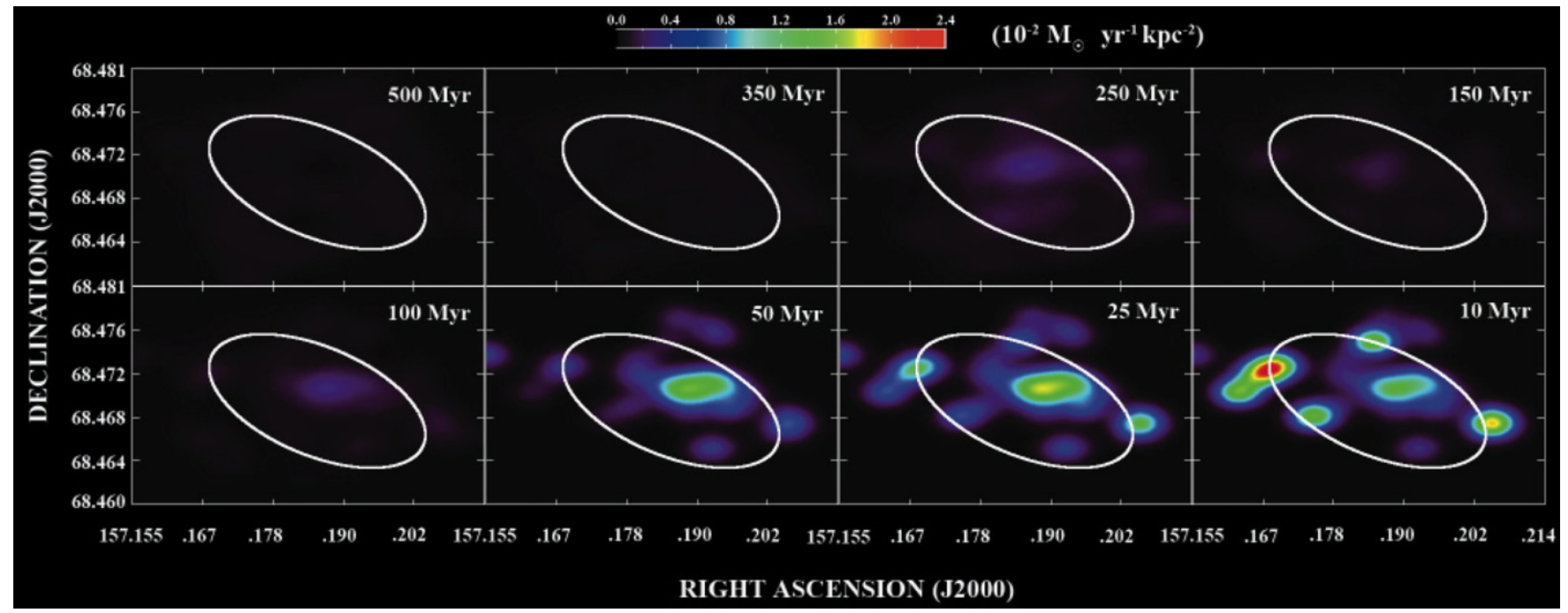

Figure 4. Selected still frames from the spatially resolved recent SFH of the SGS region. The white ellipse corresponds to the elliptical outline of the SGS itself shown in Figure 1. The spatial resolution of the images is $\sim 8^{\prime \prime}$, similar to that of the $\mathrm{H}$ I observations. The movie can be seen at http://www.astro.umn.edu/ dweisz/2574/sgs.mov.

that over the past $500 \mathrm{Myr}$, the SFR is always higher than the lifetime averaged SFR, with notable increases at $\sim 200-300 \mathrm{Myr}$ and within the last $\sim 25$ Myr. From the most recent time bin $(0-10 \mathrm{Myr})$ we see a dramatic increase in the SFR, which is in agreement with the $\mathrm{H} \alpha$ derived SFR for this region (e.g., Miller \& Hodge 1994; Cannon et al. 2005). Because the SGS region, i.e., all of panel (c) in Figure 1, is larger than the SGS itself, we tested for the possibility that SF from the additional area influenced our SFH. We measured the SFH interior to the SGS only and found differences which were deemed insignificant.

\subsection{Spatially Resolved SFH of the SGS}

BHeBs are powerful tools for studying both the ages and locations of SF from $~ 10-500$ Myr (see Dohm-Palmer et al. 1997, 2002; Weisz et al. 2008). They provide us leverage because (a) this evolutionary phase is very short relative to the main-sequence lifetime and (b) BHeBs of different ages do not overlap on the CMD (Dohm-Palmer et al. 1997; Gallart et al. 2005; Weisz et al. 2008). The result is that we can create density maps of BHeBs at different ages, which trace the spatial location of SF events over time. Inherent uncertainties in distance, extinction, photometry, and isochrones can all affect the precise ages of the BHeBs. Because of the low metallicities in dIs and the high quality of our ACS data, we find the observational related uncertainties to be small, and have calculated isochrone based errors to be $\sim 10 \%$ (Weisz et al. 2008). In addition to uncertainties in ages, the locations of the BHeBs can be influenced by proper motions of the stars. The stars we consider are thought to belong to clusters and are thus bound by gravity. Although they will not remain gravitationally bound indefinitely, the timescale for dissipation of such stellar associations is $\sim 600$ Myr to 1 Gyr (Dohm-Palmer et al. 1997), much longer than the timescale we consider for the SGS. To transform the density of BHeBs to an SFR per area, we multiply each density map by the corresponding SFR, measured using the code of Dolphin (2002), divide by the image area, and interpolate in both space and time to create a spatially resolved SFH movie. The final spatial resolution of the movie is $\sim 8^{\prime \prime}$, similar to that of the H I observations, and the movie frames are then interpolated onto a grid with 5 Myr sampling.
Figure 4 shows selected still frames from the spatially resolved SFH movie of the SGS region; the outline of the SGS is indicated by the white ellipse. We can see that in the oldest frames, 500 and $350 \mathrm{Myr}$, there is no major SF going on in the region. From 200-300 Myr, we see that there is an elevated period of SF in the center of the SGS. A period of relative quiescence lasts until $\sim 100 \mathrm{Myr}$, when an SF event begins to strengthen inside the central regions of the SGS. The peak strength of this central event is at $\sim 25 \mathrm{Myr}$, after which SF subsides centrally, but increases in intensity on the rim.

The location of the SF events in the center of the SGS and on the rim are in excellent agreement with observations from other wavelengths. The central event coincides well with UV observations by Stewart \& Walter (2000). The triggered SF on the rim is traced by $\mathrm{H} \alpha$ and Spitzer IR imaging (e.g., Cannon et al. 2005) and is in excellent agreement with our 10 Myr frame. The 200-300 Myr event is beyond the look-back time of the UV and IR fluxes, and is only traceable with the deep photometry presented here.

\section{DISCUSSION}

To understand the current state of the SGS, we consider the energetics of both the $\mathrm{H}$ I and SF, the role of triggered SF, and the timing of the events that led to the evacuation of the $\mathrm{HI}$ and secondary SF around the rim. To do this we examine the efficiency of energy, i.e., the feedback efficiency, transferred from stellar feedback into moving the ISM in the context of both an expanding and stalled shell.

Following the model of Chevalier (1974), one can compute the energy necessary to evacuate the H I mass interior to the SGS to be $\sim 2 \times 10^{53}$ erg (e.g., Walter et al. 1998; Walter \& Brinks 1999; Cannon et al. 2005). Using STARURST99 (Leitherer et al. 1999), we can independently calculate the energy associated with SF in the SGS region by inputting our SFH and assuming the same IMF as was used to measure the SFH (see Weisz et al. 2008). We computed the total cumulative kinetic energy (i.e., stellar winds and supernovae) of the SGS region over the past 500 Myr to be $\sim 3 \times 10^{55}$ erg (Figure 3 ). Because of our relatively coarse time resolution, we need to consider that our elevated SF periods, may be shorter bursts averaged out over a longer time. Thus, we used STARBURST99 to model the SFH 
as both a series of constant episodes of SF, as well instantaneous bursts for the elevated SF episodes and found the energetics to be consistent.

At first pass, it may seem that SF generates an unreasonably large amount of energy compared to the Hi energy requirement. However, by considering the feedback efficiency we can reconcile the apparent discrepancy. Assuming that each supernova (SN) imparts $10^{51} \mathrm{erg}$ in into moving the ISM we find $\mathrm{a} \sim 1 \%$ stellar feedback efficiency, which is on the low end of the spectrum that ranges from $\sim 1-20 \%$ (e.g., Theis et al. 1992; Cole et al. 1994; Padoan et al. 1997; Thornton et al. 1998). However, we have really computed a lower limit on the efficiency, as the H I energy is a lower bound, and the SF energy is an upper bound. The H I energy was computed using an assumed smooth uniform $\mathrm{HI}_{\mathrm{I}}$ distribution inside the SGS. Given that the central SF event in the SGS was extremely powerful, it is likely that there was a higher density of gas interior to the SGS in the past (e.g., Oey et al. 2002). For example, the model of Chevalier (1974) predicts that an increase in a factor of 10 in the gas density would change our feedback efficiency to $\sim 10 \%$. When we consider the energy input into the ISM due to SF, only a fraction of this energy is used to move the ISM, with the rest lost to heating and radiation. Simulations suggest $\sim 10^{50}$ erg per SN is a more realistic amount of SN energy imparted into moving the ISM (Cole et al. 1994; Thornton et al. 1998), which also increases our feedback efficiency. Finally, if the SGS has stalled expansion and broken out of the plane of the galaxy, the energy from SF simply escapes and does not go into moving the ISM. Despite the large uncertainties in the calculations of both energy quantities, we find that our feedback efficiency falls in the range of the values typically calculated from simulations, indicating the SF generated an appropriate amount of energy for creation of the SGS.

The SGS presents a clear case of not only sequential SF, but also triggered (i.e., causally connected) SF. From the spatially resolved SFH movie (Figure 4), we see clearly that the central burst predates the SF on the rim, which is in excellent agreement with age estimates in the literature (e.g., Walter et al. 1998; Cannon et al. 2005; Pasquali et al. 2008). Establishing a causal relationship between SF events is generally non-trivial and only a handful of cases of triggered SF have been established (see Oey et al. 2005 and references therein). In the case of the SGS, we have a recent SF episode interior to the SGS, which peaked $\sim 25 \mathrm{Myr}$ ago and the bulk of the recent SF on the rim is $<10 \mathrm{Myr}$ old, indicated by both the SF movie and the $\mathrm{H} \alpha$ observations. Thus, it appears that the central SF event initiated the expansion of the shell, which swept up the ISM, and when the gas on the rim becomes sufficiently dense, secondary SF began. As a slight twist to this scenario, we can speculate about the role of the older (200-300 Myr), central SF event. Because of the location of the older event and energy input into the ISM, it is possible that this older event cleared out some or all of the SGS, and the more recent event simply served to accelerate the expansion and trigger the SF around the rim. Or perhaps the older event created one spherical shell, which merged with a younger shell to account for the ellipticity of the SGS. To better our understanding of the role of both young and old SF events in reshaping the ISM, we will need to employ similar analysis techniques on a statistically significant sample of holes and shells in the ISM of nearby dIs.

Support for this work was provided by NASA through grants GO-9755 and GO-10605 from the Space Telescope Science Institute, which is operated by AURA, Inc., under NASA contract NAS5-26555. Support is also provided by NRAO, which is operated by Associated Universities, Inc., under cooperative agreement with the National Science Foundation. D.R.W. is grateful for support from a Penrose Fellowship. E.D.S. is grateful for partial support from the University of Minnesota. This research has made use of NASA's Astrophysics Data System Bibliographic Services and the NASA/IPAC Extragalactic Database (NED), which is operated by the Jet Propulsion Laboratory, California Institute of Technology, under contract with the National Aeronautics and Space Administration.

\section{REFERENCES}

Bureau, M., Walter, F., van Gorkom, J., \& Carignan, C. 2004, in IAU Symp. 217, Recycling Intergalactic and Interstellar Matter, ed. P.-A. Duc, J. Braine, \& E. Brinks (Dordrecht: Kluwer), 452

Cannon, J. M., et al. 2005, ApJ, 630, L37

Chevalier, R. A. 1974, ApJ, 188, 501

Cole, S., Aragon-Salamanca, A., Frenk, C. S., Navarro, J. F., \& Zepf, S. E. 1994, MNRAS, 271, 781

Dolphin, A. E. 2000, PASP, 112, 1383

Dolphin, A. E. 2002, MNRAS, 332, 91

Dohm-Palmer, R. C., Skillman, E. D., Mateo, M., Saha, A., Dolphin, A., Tolstoy, E., Gallagher, J. S., \& Cole, A. A. 2002, AJ, 123, 813

Dohm-Palmer, R. C., et al. 1997, AJ, 114, 2527

Gallart, C., Zoccali, M., \& Aparicio, A. 2005, ARA\&A, 43, 387

Harris, J., \& Zaritsky, D. 2008, PASA, 25, 116

Karachentsev, I. D., et al. 2002, A\&A, 383, 125

Kamphuis, J., Sancisi, R., \& van der Hulst, T. 1991, A\&A, 244, L29

Leitherer, C., et al. 1999, ApJS, 123, 3

Loeb, A., \& Perna, R. 1998, ApJ, 503, L35

Marigo, P., Girardi, L., Bressan, A., Groenewegen, M. A. T., Silva, L., \& Granato, G. L. 2008, A\&A, 482, 883

Miller, B. W., \& Hodge, P. 1994, ApJ, 427, 656

Oey, M. S., Groves, B., Staveley-Smith, L., \& Smith, R. C. 2002, AJ, 123, 255

Oey, M. S., Watson, A. M., Kern, K., \& Walth, G. L. 2005, AJ, 129, 393

Padoan, P., Jimenez, R., \& Jones, B. 1997, MNRAS, 285, 711

Pasquali, A., et al. 2008, ApJ, 687, 1004

Puche, D., Westpfahl, D., Brinks, E., \& Roy, J.-R. 1992, AJ, 103, 1841

Rhode, K. L., Salzer, J. J., Westpfahl, D. J., \& Radice, L. A. 1999, AJ, 118, 323

Skillman, E. D., Terlevich, R., Teuben, P. J., \& van Woerden, H. 1988, A\&A, 198,33

Skillman, E. D. (ed.) 1996, in ASP Conf. Ser. 106, The Minnesota Lectures on Extragalactic Neutral Hydrogen (San Francisco, CA: ASP), 208

Stewart, S. G., \& Walter, F. 2000, ApJ, 529, 201

Tenorio-Tagle, G., \& Bodenheimer, P. 1988, ARA\&A, 26, 145

Theis, C., Burkert, A., \& Hensler, G. 1992, A\&A, 265, 465

Thornton, K., Gaudlitz, M., Janka, H.-T., \& Steinmetz, M. 1998, ApJ, 500, 95

van der Hulst, J. M. 1996, in ASP Conf. Ser. 106, The Minnesota Lectures on Extragalactic Neutral Hydrogen, ed. E. D. Skillman (San Francisco, CA: ASP), 47

Walter, F., \& Brinks, E. 1999, AJ, 118, 273

Walter, F., Kerp, J., Duric, N., Brinks, E., \& Klein, U. 1998, ApJ, 502, L143

Weisz, D. R., Skillman, E. D., Cannon, J. M., Dolphin, A. E., Kennicutt, R. C., Jr., Lee, J. C., \& Walter, F. 2008, ApJ, 689, 160 